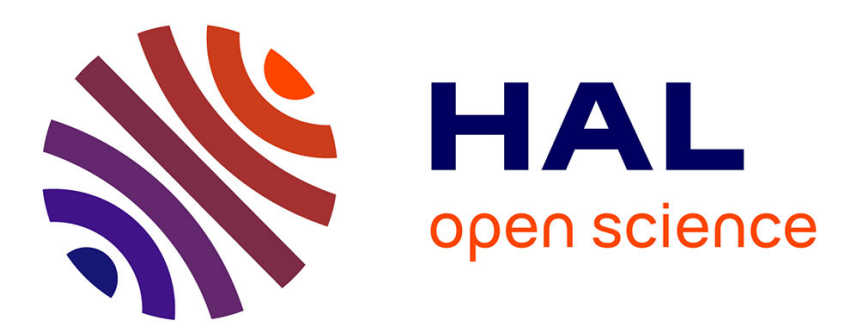

\title{
Glass-forming ability of elemental zirconium
}

Sébastien Becker, Emilie Devijver, Rémi Molinier, Noël Jakse

\section{To cite this version:}

Sébastien Becker, Emilie Devijver, Rémi Molinier, Noël Jakse. Glass-forming ability of elemental zirconium. Physical Review B, 2020, 102 (10), pp.104205. 10.1103/PhysRevB.102.104205 . hal03181321

\section{HAL Id: hal-03181321 \\ https://hal.science/hal-03181321}

Submitted on 25 Mar 2021

HAL is a multi-disciplinary open access archive for the deposit and dissemination of scientific research documents, whether they are published or not. The documents may come from teaching and research institutions in France or abroad, or from public or private research centers.
L'archive ouverte pluridisciplinaire HAL, est destinée au dépôt et à la diffusion de documents scientifiques de niveau recherche, publiés ou non, émanant des établissements d'enseignement et de recherche français ou étrangers, des laboratoires publics ou privés. 


\title{
Glass forming ability of elemental zirconium
}

\author{
Sébastien Becker ${ }^{1,2}$, Emilie Devijver², Rémi Molinier ${ }^{3}$, and Noël Jakse ${ }^{1}$ \\ ${ }^{1}$ Univ. Grenoble Alpes, \\ CNRS, Grenoble INP, SIMaP, \\ F-38000 Grenoble, France \\ ${ }^{2}$ Univ. Grenoble Alpes, \\ CNRS, Grenoble INP, LIG, \\ F-38000 Grenoble, France \\ ${ }^{3}$ Univ. Grenoble Alpes, CNRS, \\ Grenoble INP, Institut Fourier, \\ F-38000 Grenoble,France
}

(Dated: September 9, 2020)

\begin{abstract}
We report large-scale molecular dynamics simulations of the glass formation from the liquid phase and homogeneous nucleation phenomena of pure zirconium. For this purpose, we have built a modified embedded atom model potential, in order to reproduce relevant structural, dynamic and thermodynamic properties from $a b$ initio and experimental data near the melting point. By means of liquid-solid interface simulations, we show that this potential provides a thermodynamic melting temperature and densities of the solid and liquid state in good agreement with experiments. Using melt-quenching simulations with one million atoms, we determine the glass transition from the temperature evolution of the inherent structure energy as well as the nose of the time-temperature-transformation curve located in the deep undercooling regime. We identify the local structural origin of the glass forming ability as a competition between bcc and five-fold polytetrahedral structures that may represent an impediment of rapid homogeneous nucleation at such high undercoolings. This suggests the ability of single elemental zirconium to form a glass from the melt with cooling rates of at least $10^{12} \mathrm{~K} / \mathrm{s}$ compatible with modern experiments.
\end{abstract}




\section{INTRODUCTION}

The possibility to form a metallic glass $(\mathrm{MG})$ from the melt was reported for the first time [1] in the 60's by splat quenching of a eutectic Au-Si alloy with cooling rates as high as $10^{6} \mathrm{~K} / \mathrm{s}$. This has triggered fundamental questions regarding the possibility of forming a single-element metallic glass by cooling the melt at a sufficiently high cooling rate to avoid crystallization. Subsequent attempts for pure nickel by splat-quenching with cooling rate of $10^{10} \mathrm{~K} / \mathrm{s}$ showed an amorphous state with a crystallization temperature of $425 \mathrm{~K}$ higher than the room temperature [2], but it was suspected to be stabilized by impurities such as oxygen or carbon. Earlier studies using quench condensation on substrates [3] as well as other techniques [4] seemed to concur with the impossibility to form a metallic glass with a single element. Later, atomized iron droplets of about $30 \mathrm{~nm}$ in size were however produced $[5,6]$ with mixed crystal and amorphous structure at large undercoolings with cooling rates of $10^{7} \mathrm{~K} / \mathrm{s}$. It was further shown that a monoatomic glass is more easily formed from bodycentered cubic (bcc) than for face-centered cubic (fcc) or hexagonal closed-packed (hcp) metals [6]. This finding for bcc metals was further confirmed very recently on nanorods with cooling rates as large as $10^{14} \mathrm{~K} / \mathrm{s}$ [7]. This implies that homogeneous crystal nucleation for such large undercooling is impeded in the case of droplets while the growth might be suppressed in the case of the nanorods as they were attached to their crystalline ends [8].

As crystal nucleation is essentially initiated by heterogeneous nucleation from impurities, surfaces, or near grain boundaries [9], homogeneous crystal nucleation is driven by complex phenomena [10] difficult to capture experimentally [11] and thus remains a challenging issue. Despite similarities of the liquid and the crystal in their density and coordination number, the seminal work by Turnbull [12] on undercooled metallic melts demonstrated that significant undercooling can be obtained. This implied that nucleation is an activated process in which the liquid transforms to the crystal by overcoming a free energy barrier. This is possible only if the liquid possesses an atomic structure substantially different from that of a simple crystal, and orders in the vicinity of a crystal surface [13]. It was then suggested by Frank [14] that the energy minimization in a monoatomic liquid favors the formation of local icosahedral structure. Such a five-fold symmetry, predominantly built from slightly distorted tetrahedral structures, is incompatible with simple closed-packed lattices such as the bcc, fcc and hcp crystals that satisfy a global energy minimum [15]. 
Investigations of the interplay between homogeneous nucleation and local polymorphism [16] remain experimentally challenging to observe [11, 17-22], especially at large undercoolings $\Delta T=T-T_{M}, T$ being the temperature of the studied liquid and $T_{M}$ its melting or liquidus temperature. They were often carried out using direct particle based simulation methods like molecular dynamics (MD) or Monte-Carlo (MC) [23, 24]. For Al, a prototype fcc metal, nucleation might occurs primarily by formation of fcc nuclei along with hep defects $[25,26]$. The situation is less clear for $\mathrm{Cu}$ since former MD simulations [27] showed an initial stage of nucleation with a fcc local ordering while very recent ones [28] involve metastable bcc polymorph pre-critical nuclei. For Fe and $\mathrm{Mg}$, early stages of nucleation involve formation of respectively bcc and hep phase corresponding to their stable phase. In $\mathrm{Na}$ [29], another bcc metal, it was shown that the supercooled liquid, with significant icosahedral and defective icosahedral local order, transforms during the nucleation process into the stable bcc structure via defective bcc local structure. Such a competition between the bcc and icosahedral local orderings in the undercooled liquid was observed in Zr [30, 31] and Ta [32] by means of ab initio molecular dynamics (AIMD) simulations. However, for these two metals, it was shown that the structural ordering can be interpreted on the basis of a competition between a polytetrahedral local order, more complex that the simple icosahedral one, and a bcc-type ordering. For Zr, the bcc-type ordering increases with the degree of undercooling together with more numerous Frank-Kasper (FK) polyhedra such as Z14, Z15, and Z16 [33]. A similar situation occurs for Ta but with a local order close to that of the A15 phase.

Complex polytetrahedral orderings found in some bcc elemental undercooled liquid metals [30-32] might therefore play a significant role in the frustration against crystallization scenario suggested by Tanaka $[34,35]$ when approaching the glass transition temperature $T_{G}$. Interestingly, for pure Ta, formation of a stable glass was observed [7] that could be a result of this concept of frustration. However, the situation is less clear for Zr since attempts to form glassy spheres using atomized droplets [5, 6] failed and formation of a metallic glass at high pressure [36] was subject to misinterpretation of the experiments [36, 37]. Therefore, the question of the existence of a stable amorphous state for Zr remains open.

The aim of the present work is therefore to study the glass formation as well as the onset of crystallization features of pure Zr as a function of the degree of undercooling $\Delta T$ in the range between $T_{M}$ and $T_{G}$, by means of large-scale MD simulations [38] up to one million 
atoms. For this purpose we have designed a semi-empirical potential within the framework of the Modified Embedded Atom Model (MEAM) [39] based on a bcc reference structure, which reproduce the structural and thermodynamic features of the liquid state and solid $\beta$-phase in the vicinity of the melting point. We determined the glass transition temperature and timetemperature transformation (TTT) diagram at large undercoolings. Our findings indicate that elemental Zr might form a metallic glass with cooling rates higher than $10^{12} \mathrm{~K} / \mathrm{s}$, now being achievable experimentally [7], and results from a strong competition between bcc and fivefold FK polytetrahedral local structural orderings, impeding homogeneous nucleation.

The layout of the paper is the following. Section II is devoted to the technical aspects of the simulations and the design of the potential. Section III presents and discusses the optimization and tests of the potential as well as the results of the simulations. Finally in Section IV we draw our conclusions.

\section{SIMULATION BACKGROUND}

\section{A. Modified Embedded Atom Model}

Several semi-empirical interatomic potentials have been developed for atomistic simulations for pure Zr including second-moment tight-binding method [40], Embedded Atom Model (EAM) [41], MEAM [39, 42, 43], Reax Force Field [44] as well as Machine-Learning technique within the Kernel-Ridge Regression [45]. However, only some of then were designed to take into account the martensitic hcp-bcc transformation at ambient pressure $[40,41,45]$. Moreover, none of them were designed to take into account the liquid state properties explicitly and specifically local structural properties, self-diffusion and the melting temperature. In the context of the present work, i.e. the study of the liquid and undercooled properties of Zr, our strategy is then to develop a MEAM potential using a bcc reference structure which corresponds to the underlying high temperature crystalline structure ( $\beta$-phase). In the original MEAM potential, many-body and directional bonding aspects are taken into account in the first neighborhood, but here we use its second nearest-neighbor $(2 \mathrm{NN})$ extension [46] in order to take larger varying atomic environment into account. Considering a system of $N$ atoms, the potential energy functional in the 
MEAM formalism can be expressed in a general form as

$$
E=\sum_{i=1}^{N}\left[F_{i}\left(\rho_{i}\right)+\frac{1}{2} \sum_{j \neq i=1}^{N} \Phi\left(r_{i j}\right)\right],
$$

where $F_{i}\left(\rho_{i}\right)$ is the embedding energy function depending on the background electronic density $\rho_{i}, \Phi\left(r_{i j}\right)$ is the pair potential interaction as a function of interatomic distance $r_{i j}$ between atoms $i$ and $j$. The MEAM formalism is well documented in the literature, therefore we refer the reader to Refs. [46] and [43] for a detailed description.

The electron density is calculated first for each atomic site from an analytic expression taking into account the length and directionality in bonding of the neighbors [46], and the background electron density is taken in the form $2 \rho_{i} /(1+\exp (\Gamma))$, where $\Gamma$ is electronic density expansion over the $t^{(k)}(k=0, \ldots, 3)$ electronic structure parameters. Then from a specific form of the embedding function and the total energy, the pair potential $\Phi(r)$ in Eq. (1) is estimated using the equation of state of Rose [48]

$$
E_{\text {Rose }}=-E_{\text {coh }}\left(1+a^{*}+d a^{* 3}\right) e^{-a^{*}}
$$

where $a^{*}=\alpha\left(r / r_{e}-1\right)$ where $r_{e}$ is the nearest-neighbor distance in the bcc reference structure and $\alpha=\left(9 B \Omega / E_{c o h}\right)^{1 / 2}, B$, being the bulk modulus, $E_{c o h}$ the cohesive energy, and $\Omega$ the atomic volume. The $2 \mathrm{NN}$ part is controlled through the screening parameters $C_{\min }$ and $C_{\max }$, with $C_{\min }$ taking values below 2 , and we follow strictly the original paper by Lee $e t$ al. [46] which is implemented in LAMMPS [38]. Accordingly, the cutoff radius of the potential takes a value of $7.60 \AA$.

From a previous attempt [47], we kept the electronic structure parameters $\beta^{(k)}$ and $t^{(k)}$ $(k=0, \ldots, 3)$ and optimized the parameters $r_{e}, d, C_{\min }$, and $C_{\max }$ only. The value of $r_{e}$ was adjusted to reproduce at best the density of the liquid and bcc crystalline phase, and $d$ was determined to get at best the experimental melting temperature of $\mathrm{Zr}$. It is worth mentioning that $d$ is usually fitted on the pressure variation of the bulk modulus in the solid phase $(\partial B / \partial P)$. However, the amplitude of variation of $d$ considered here should not alter much this quantity [43] while having a significant impact on melting, as it affects essentially on the well depth of the pair potential $\phi\left(r_{i j}\right)$ (see Eq. 2). The screening parameters $C_{\min }, C_{\max }$ were fitted to get the better representation of at least the two first peaks of the pair correlation function obtained from the AIMD simulation (see Sec. II B). Interestingly enough, the values of the hcp version of the MEAM potential obtained by Kim 


\begin{tabular}{cccccccccccccc}
\hline \hline$E_{\text {coh }}(\mathrm{eV})$ & $r_{e}(\AA)$ & $\alpha$ & $A$ & $\beta^{(0)}$ & $\beta^{(1)}$ & $\beta^{(2)}$ & $\beta^{(3)}$ & $t^{(1)}$ & $t^{(2)}$ & $t^{(3)}$ & $C_{\min }$ & $C_{\max }$ & $d$ \\
\hline 6.29 & 3.10 & 4.27 & 0.72 & 4.10 & 1.00 & 1.00 & 1.00 & 5.80 & -0.350 & -1.30 & 1.00 & 1.44 & -0.069 \\
\hline \hline
\end{tabular}

TABLE I: Parameters of MEAM potentials (see text for their definition).

et al. [43] $C_{\text {min }}$, and $C_{\max }$ correspond to the best compromise. It should be also noted that the structural energy difference $\Delta E_{h c p \rightarrow b c c}=0.017 \mathrm{eV}$ is consistent with the value obtained by them [43] and similar values of the mechanical properties than those reported previously [47] are found.

\section{B. Ab initio molecular dynamics}

In order to provide reference values for the pair-correlation function and self-diffusion coefficient for the MEAM potential fitting, AIMD simulations have been performed using the VASP code $[49,50]$. The projected augmented-wave (PAW) method was used to describe the electron-ion interaction $[51,52]$ and the exchange-correlation energy was taken from the generalized gradient approximation in the PBE form [53]. In the present PAW potential, the $5 \mathrm{~s}, 4 \mathrm{~d}$ orbitals as well as the semi-core $4 \mathrm{~s}$ and $4 \mathrm{p}$ orbitals are treated as valence orbitals with a plane-wave cutoff of $300 \mathrm{eV}$. Only the $\Gamma$-point has been considered to sample the supercell Brillouin zone.

The simulations were carried out in the NVT ensemble (constant volume $V$, temperature $T$, and number of particles $N$ ), with a Nosé thermostat [23, 24], similarly than in our previous work [30] but using $N=256$ atoms in order to have a more refined description of the structure and dynamics in the liquid state. Equations of motions were solved using Verlet's algorithm in the velocity form with a time step of 1 fs. The volume $V$ of the cell was fixed to reproduce the experimental densities [61] and was subject to standard periodic boundary conditions. We have considered $T=2500 \mathrm{~K}$, well above the experimental melting temperature $T_{M, \exp }=2128 \mathrm{~K}$. The simulation was started from an initial configuration with random positions and equilibrated at $T=2500 \mathrm{~K}$ for $10 \mathrm{ps}$. The run was continued for 30 ps to calculate the pair-correlation function, and the velocity auto-correlation function. 


\section{Classical molecular dynamics}

All MD simulations were performed with the LAMMPS code [38] in the isobaricisothermal ensemble (constant temperature, pressure and number of particles) with ambient pressure, using the Nose-Hoover thermostat and barostat $[23,24]$ with periodic boundary conditions $(\mathrm{PBC})$ in the three directions of space. As for AIMD, Verlet's algorithm in the velocity form was used for the numerical integration of the equations of movements, with a time step of 2 fs. We conducted two types of simulations whose characteristics are described in Table II: (i) MD with $N \simeq 128000$ atoms of liquid-solid interfaces (LSI) for the purpose of determining the melting temperature by the two-phase coexistence technique with different crystalline orientations; (ii) melt-quenching simulations of bulk systems with $N=5488$, 16000 or 1024000 atoms down to the glass transition temperature as well as simulation of isotherms in the undercooled region to determine the time-temperature-transformation curves from homogeneous nucleation events.

The liquid-solid interface simulations are firstly carried out for the determination of the melting temperature $T_{M}$ of the MEAM potential. The procedure is similar to the two-phase coexistence approach formerly introduced by Morris et al. [54, 55] and slightly improved subsequently by Sun et al. [56]. A simulation cell containing around $N=128000$ atoms was set up with an initial crystalline configuration with PBC applied to the three directions of space. The crystal is heated and equilibrated at constant pressure to a temperature $100 \mathrm{~K}$ below a guess of $T_{M}$. We observed that the system remained fully crystalline. Half of the simulation cell is subsequently heated and maintained at a temperature approximately 1500 $\mathrm{K}$ above $T_{M}$ until a complete melting was observed. The liquid part was then cooled down and equilibrated at a temperature $100 \mathrm{~K}$ above the estimated $T_{M}$. This procedure creates a coexisting solid-liquid simulation cell containing two crystal-melt interfaces due to the PBC. The simulation of the entire system is pursued in the isobaric-isoenthalpic ensemble in which the simulation box was allowed to change its length only along the $x$ direction in order to keep a zero pressure. As a result, the temperature of the coexisting liquid-solid interface evolved toward a steady state corresponding to the thermodynamic melting temperature. The simulation is continued for $1 \mathrm{~ns}$, and the average melting temperature is determined on the last $100 \mathrm{ps}$ if a steady position of the two interfaces is observed. If a complete melting or solidification occurs, the procedure is started over again with a refined guess of 


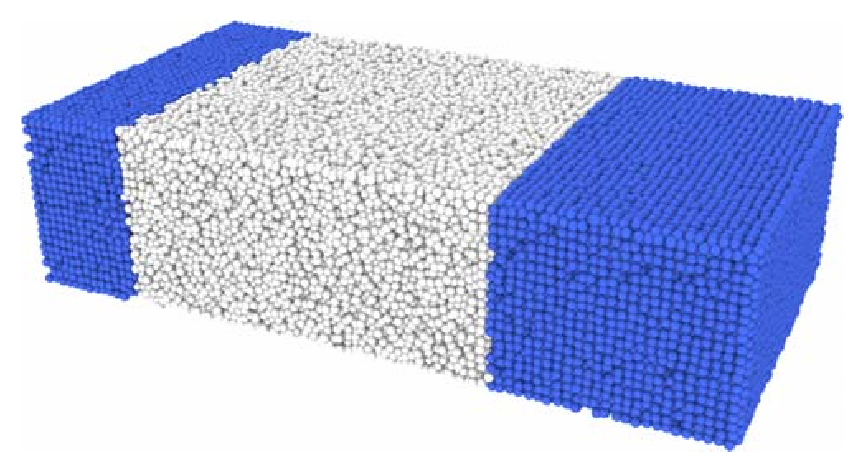

FIG. 1: Upper panel: snapshot of the liquid-solid (100) interface with $640 \times 10 \times 10$ shape; Lower panel: snapshot of the liquid-solid (100) interface with $80 \times 40 \times 20$ shape.

the melting temperature. This procedure is applied for three crystalline orientations, i.e. (100), (111), and (110) whose characteristics are described in Table II. Two box shapes are also considered for the (100) interface for which a snapshot obtained with the OVITO software [57] is shown in Figure 1.

For the melt quenching of the bulk systems, simulations were started at a temperature $T=2500 \mathrm{~K}$ from an initial configuration with random atomic positions. After equilibration at this temperature, the system was cooled down to $300 \mathrm{~K}$ with a cooling rate of $10^{12}$ $\mathrm{K} / \mathrm{s}$. During this process, thermodynamic properties are calculated and configurations are recorded each 1000 time steps, for the purpose of starting subsequent MD simulations along chosen isotherms in the undercooled and glassy states. It is worth mentioning that for Bulk 2 system, various cooling rates were explored, namely $10^{13}, 10^{12}$, and $10^{11} \mathrm{~K} / \mathrm{s}$. While for the two faster cooling rates, homogeneous nucleation can be avoided, for $10^{11} \mathrm{~K} / \mathrm{s}$ crystallisation during cooling was observed, guiding our choice for the cooling rate with Bulk 3 system to study the homogeneous nucleation process.

The average position location of each solid-liquid interface and the local structural anal- 


\begin{tabular}{llclll}
\hline \hline & $N$ & Shape & $T_{M}(\mathrm{~K})$ & $\rho_{M}^{L}(\mathrm{~K})(\AA)^{-3}$ & $\rho_{M}^{S}(\mathrm{~K})(\AA)^{-3}$ \\
\hline Bulk 1 & 5488 & $14 \times 14 \times 14$ & & & \\
Bulk 2 & 16000 & $20 \times 20 \times 20$ & & & \\
Bulk 3 & 1024000 & $80 \times 80 \times 80$ & & & \\
Interface 1 (100) & 128000 & $640 \times 10 \times 10$ & $2119 \pm 15$ & $0.0371 \pm 0.03$ & $0.0386 \pm 0.01$ \\
Interface 2 (100) & 128000 & $80 \times 40 \times 20$ & $2111 \pm 15$ & $0.0377 \pm 0.01$ & $0.0388 \pm 0.01$ \\
Interface 1 $(110)$ & 127960 & $640 \times 10 \times 10$ & $2132 \pm 15$ & $0.0373 \pm 0.01$ & $0.0386 \pm 0.04$ \\
Interface 1 (111) & 128016 & $640 \times 10 \times 10$ & $2114 \pm 15$ & $0.0377 \pm 0.03$ & $0.0381 \pm 0.01$ \\
\hline \hline
\end{tabular}

TABLE II: Parameters of classical molecular dynamics simulations for bulk and solid -liquid interface. $N$ stands for the number of atoms and the shape of the simulation box is given in units of primitive bcc cells in the $x, y$, and $z$ direction. $T_{M}, \rho_{M}^{L}$ and $\rho_{M}^{S}$ are respectively the calculated melting temperature, liquid and solid densities for the different interfaces. The uncertainty of the melting temperature is taken as the standard deviation with a confidence interval of $95 \%$ over the four estimations of the melting temperature.

ysis were determined using, as a descriptor, the common-neighbor analysis (CNA) [58] with the indexing of Faken and Jonsson [59] and a bond-based algorithm as implemented in the OVITO software [57] where a uniform cut-off radius corresponding to the first minimum of the pair-correlation function of the liquid is applied to create bonds between pairs of particles. The CNA classifies pairs around each atom by sets of three indices: the first index represents the number of near neighbors common to this pair, the second index corresponds to the number of nearest-neighbor bonds among the shared neighbors, and the third index indicates the longest chain of bonded atoms among them. For instance, 421 and 422 bonded pairs are characteristic of close packed structures fcc and hcp, respectively. The occurrence of 444 and 666 pairs, with specific proportions, signals the presence of bcc ordering. The degree of five-fold symmetry is obtained from the proportion of 555, 554 and 433 pairs, which represent perfect (555) and distorted FFS based motifs. The system at the interface is considered as solid at a given distance if at least $50 \%$ of the atoms have a local environment characterized as bcc according to the CNA. 


\section{RESULTS AND DISCUSSION}

\section{A. Optimization of the MEAM potential}

In a first stage, the optimization of the parameters $d, r_{e}, C_{\min }$, and $C_{\max }$ was carried out to reproduce simultaneously at best the experimental melting temperature, the density of the liquid and bcc solid phase, and the two first peaks of the pair-correlation function, respectively. Table II gathers the values of melting temperature determined from the liquidsolid interface for the three crystalline orientations and the two box shapes (see Fig. 1). All the values are mutually compatible indicating that shape and crystalline orientation effect are essentially negligible. Finally, $T_{M}=2119 \mathrm{~K}$ was accepted as the average of the 4 values determined, which is in very good agreement with the experimental one, namely $2128 \mathrm{~K}$. It should be mentioned that a better adjustment could have been obtained, but $d$ has also a correlated effect together with $r_{e}$ on the density and a reasonable compromise between the two physical quantities had to be made.

The liquid and solid densities at melting, extracted respectively from the liquid and solid parts of each LSI simulations, are given in Table II. Again, essentially no shape and orientation effect is seen. The various measurements of the liquid densities and their temperature evolution span over a period of 7 decades and were reviewed recently [60, 61]. They display a significant departure of $13 \%$ between $0.0363 \AA^{-3}$ and $0.0412 \AA^{-3}$. Our simulated liquid densities at melting are close to the most recent experimental value of $0.0396 \AA^{-3}$ measured by means of electrostatic levitation technique by Wang et al. [61], with a departure of $4.5 \%$. The density of the bcc solid at melting compare well with the experimental data of $\mathrm{Lu}$ et al. [62] with underestimation of $4 \%$ only. Interestingly, solidliquid density difference in the present simulations is $\Delta \rho_{S-L}=0.007 \AA^{-3}$, close to the only existing consistent measurements of Paradis and Rhim [63] giving $0.006 \AA^{-3}$.

The values of the screening parameters $C_{m i n}$ and $C_{m a x}$ are optimized at the microscopic level on the pair-correlation function, $g(r)$, in the liquid state. Taking the AIMD curve obtained in the present work at $2500 \mathrm{~K}$ as a reference, they have been obtained by minimizing the least-square deviation. The function $g(r)$ gives the probability of finding a particle $j$ at distances $r$ relative to a particle $i$ located at the origin, and reads:

$$
g(r)=\frac{N}{V} \frac{n(r)}{4 \pi r^{2} \Delta r}
$$



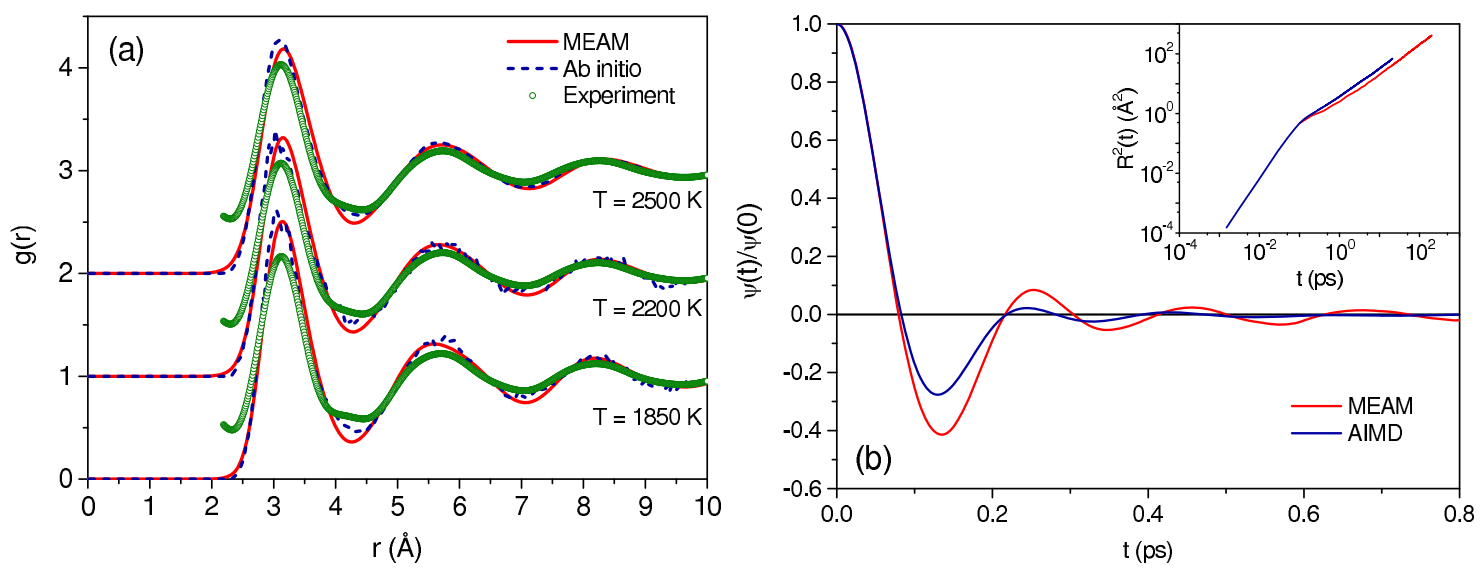

FIG. 2: (a) Pair-correlation functions at $T=2500 \mathrm{~K}, T=2200 \mathrm{~K}$, and $T=1850 \mathrm{~K}$, from the MEAM potential, AIMD of the present work as well as from the previous one [30], and compared to the experiment [64]; (b) Velocity-autocorrelation at function at $T=2500 \mathrm{~K}$ from the MEAM potential, AIMD of the present work.

$n(r)$ represents the mean number of particles $j$ in a spherical shell of radius $r$ and thickness $\Delta r$ centered on particle $i$. Integrating $g(r)$ up to their first minimum gives access to the partial coordination numbers. The comparison with AIMD simulations and existing experimental [64] data are shown in Fig. 2(a). For $T=2200 \mathrm{~K}$ and $T=1850 \mathrm{~K}$ the AIMD curves are taken from our previous work [30]. A good agreement is found with AIMD simulations indicating that the functional form given by Eq. (1) in the framework of the 2NN-MEAM [46] is able to reproduce microscopic structural aspects of $\mathrm{Zr}$ in the liquid state and their temperature evolution in the undercooled region. Both classical and ab initio MD simulations show a reasonable agreement with the experimental data [64], especially the position of the first peak and subsequent oscillations indicating that the bond length is well reproduced. Taking a cutoff radius of $4.31 \AA$. the resulting coordination number is 12.4 at $T=2500 \mathrm{~K}$ which is a bit higher than experimental value of 11.9 at $2290 \mathrm{~K}$ but still slightly lower than the $a b$ initio value of 13 . The departure being less than one atom, the coordination number obtained for the MEAM potential is deemed reasonable.

\section{B. Tests of the MEAM potential}

The reliability of the potential can be tested on the dynamics as well as some thermodynamic quantities. As for the short-time dynamics, we have considered the velocity 
auto-correlation function (VACF) :

$$
\Psi(t)=\frac{1}{N}\left\langle\sum_{i=1}^{N}\left[\mathbf{v}_{i}\left(t+t_{0}\right) \cdot \mathbf{v}_{i}\left(t_{0}\right)\right]^{2}\right\rangle_{t_{0}},
$$

where $\mathbf{v}_{i}(t)$ denotes the velocity of atom $i$ at time $t$. The angular brackets correspond to an averaging over time origins $t_{0}$. Fig. 2(b) displays the velocity auto-correlation function normalized to their value at time $t=0, \Psi(t=0)$, determined from the MEAM potential and compared to the AIMD results at $T=2500 \mathrm{~K}$. Both curves show a negative well at short time, characteristic of a back-scattering effect due to the first neighbor atoms shell, so-called cage effect, followed by a rapid damping towards zero, attesting the relaxation of the system on the sub-picosecond time scale. The cage effect is more pronounced for the MEAM, leading to a lower diffusion, however its characteristic time is similar to the AIMD. The self-diffusion coefficient $D$ is determined from the linear behavior at long times of the mean-square displacement written as

$$
R^{2}(t)=\frac{1}{N} \sum_{l=1}^{N}\left\langle\left[\mathbf{r}_{l}\left(t+t_{0}\right)-\mathbf{r}_{l}\left(t_{0}\right)\right]^{2}\right\rangle_{t_{0}}
$$

where $\mathbf{r}_{l}(t)$ denotes the position of atom $l$ at time $t$. The long time behavior of $R^{2}(t)$ is linear, which is a characteristic feature of the liquid state, as shown in the Inset of Fig. 2(b). For the MEAM potential $D=0.34 \pm 0.07 \AA^{2} / \mathrm{ps}$, which underestimates the AIMD value, i.e., $D=0.53 \pm 0.1 \AA^{2} /$ ps but still remains in reasonable agreement.

Turning now to the thermodynamics, we consider the enthalpy which is directly calculated from positions and velocities during the MD simulation by

$$
H(P, T)=\frac{1}{2} \sum_{i=1}^{N} m \mathbf{v}_{i}^{2}+\frac{1}{2} \sum_{i \neq j=1}^{N} u\left(r_{i j}\right)+P_{e x t} V,
$$

where $P_{\text {ext }}$ is the pressure imposed to the simulation box, and the volume $V$ is a dynamical variable, $m$ being the mass of the atoms. The heat capacity at constant pressure $C_{P}$ is then determined from a numerical derivative of the enthalpy using its standard definition:

$$
C_{P}(T, P)=\frac{\partial H(T, P)}{\partial T}
$$

The latent heat of fusion, also called the enthalpy of melting is an important quantity for solidification phenomena. The latter can be determined from the enthalpy difference between the liquid and solid branches at the melting temperature $T_{M}$. The enthalpy curves 
of $\mathrm{Zr}$ were determined at ambient pressure for the solid and liquid branches of the MEAM potential using MD simulation with Bulk 1 system (see Table II), and are drawn in Fig. 3. The simulation is started at $T=300 \mathrm{~K}$ with a perfect bec crystal. A simulation is performed during 100 ps (50 ps equilibration and 50 ps production) and followed by a step-wise heating with a temperature step of $100 \mathrm{~K}$ which amounts to an average heating rate of $10^{12} \mathrm{~K} / \mathrm{s}$. The average value of the enthalpy is calculated from the production stage and error bars are evaluated from the standard deviation of the Gaussian distribution. The production time along the isotherms is not long enough to observe a transition bcc-hcp transition below the experimental martensitic transition at $1139 \mathrm{~K}$, which occurs on the 10 second time scale as shown in recent electrostatic levitation measurements [65]. The procedure is repeated until a dynamic melting is observed in the simulation box, which occurred at $T=2400 \mathrm{~K}$, significantly higher than the thermodynamic melting temperature $T_{M}$ determined from LSI simulations due to overheating effects. For the liquid branch, the simulations are started at $T=3400 \mathrm{~K}$ with a disordered initial configuration.

The same procedure as for the solid branch is followed but with a step-wise cooling down to $300 \mathrm{~K}$. Above $T=2400 \mathrm{~K}$, the difference in the enthalpy from the heating and cooling processes is negligible, indicating that there is no reminiscence of the crystalline state. The cooling procedure for the liquid branch is repeated with Bulk 3 system and a cooling rate of $10^{12} \mathrm{~K} / \mathrm{s}$, and as can be seen on Fig. 3, no size effect can be detected. From the liquid and solid branches we find an enthalpy of melting of $18.2 \mathrm{~kJ} / \mathrm{mol}$ which compares quite well with the assessed experimental value [66] of $15.6 \mathrm{~kJ} / \mathrm{mol}$. Taking the numerical derivative of solid branch yields a value of $C_{P}$ at the melting point of $34.4 \mathrm{~J} / \mathrm{mol} / \mathrm{K}$ which is in very good agreement with the experimental value of $35.7 \mathrm{~J} / \mathrm{mol} / \mathrm{K}$. For the liquid a value of $34.5 \mathrm{~J} / \mathrm{mol} / \mathrm{K}$ is obtained which understimate the experimental data of $39.7 \mathrm{~J} / \mathrm{mol} / \mathrm{K}$ [66] and of $39.9 \mathrm{~J} / \mathrm{mol} / \mathrm{K}[63]$.

All these results bring us to the conclusion that the present optimized MEAM potential is reliable to treat the structural, dynamic and thermodynamic properties of $\mathrm{Zr}$ at ambient pressure. 


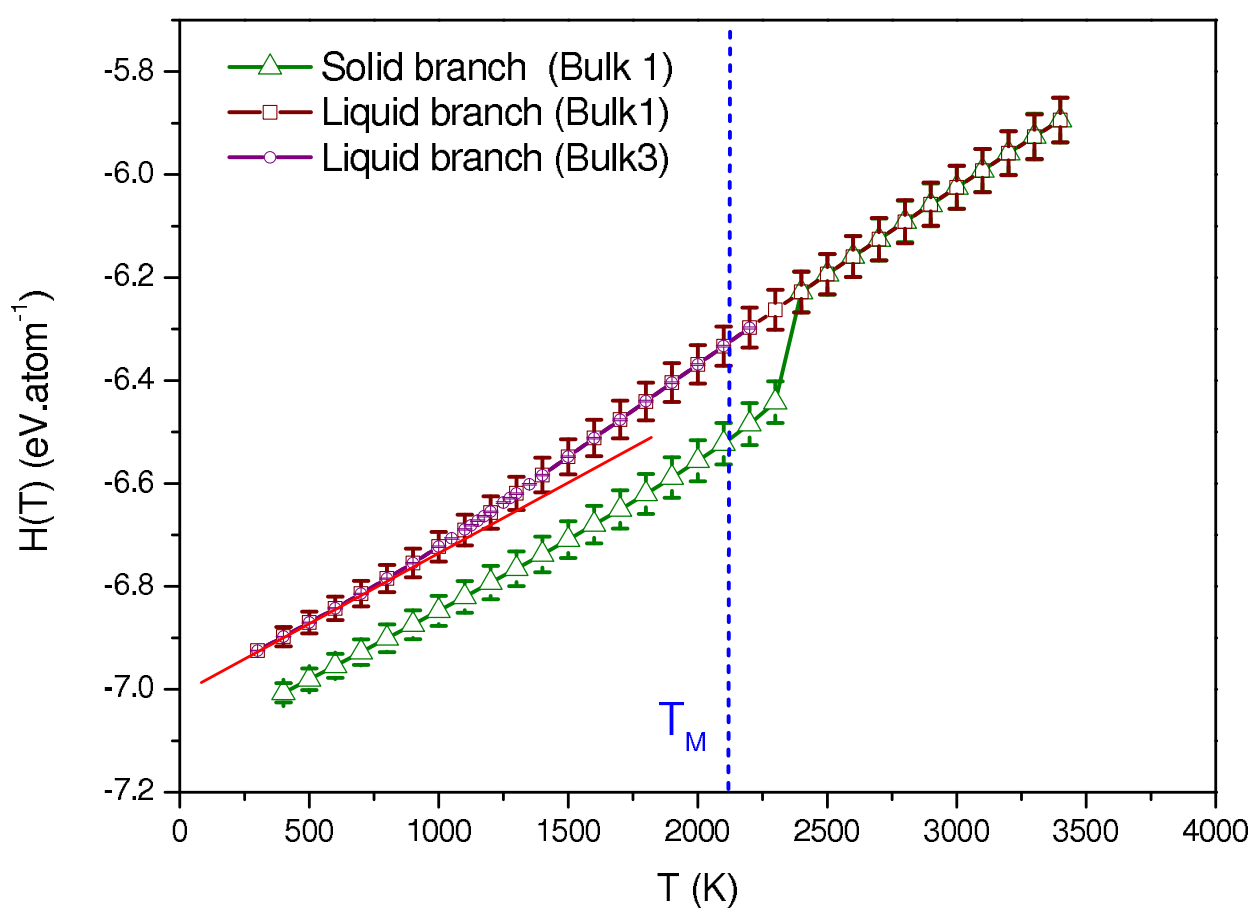

FIG. 3: Enthalpy as a function of temperature for the solid and liquid branches (see text for details). The red line marks the slope of the glassy states as a guide for the eyes for the crossover between the liquid and glassy regimes.

\section{Glass transition temperature}

The melt-quenching simulations described in Section III B and shown in Fig. 3 reveal that with a cooling rate of $10^{12} \mathrm{~K} / \mathrm{s}$ crystallization can be avoided. The enthalpy $H(T)$ exhibit a change of slope around $1000 \mathrm{~K}$ that signals that the glass transition sets in. It is worth mentioning that similarly Mo et al. [67], using an EAM potential [68] from a highly optimized ab initio procedure [69], could form a glass at a cooling-rate of $5 \times 10^{12}$ with a glass transition in the same range of temperatures as in the present work. Due to the fact that the glass transition depends on the cooling rate $[70,71]$, the temperature at which it is observed, so-called fictive temperature, is generally higher that the genuine value of the glass transition temperature, $T_{G}$. A more refined value of $T_{G}$ can be obtained based on the concept of Potential Energy Landscape (PEL) [72] from the evolution of the inherent structure energy (ISE) [73] as a function of temperature, which is displayed in Fig. 4. For this purpose, atomic configurations were recorded at the interval of $100 \mathrm{~K}$ during the quench run of Bulk 3 system at $10^{12} \mathrm{~K} / \mathrm{s}$ (from $4000 \mathrm{~K}$ to $300 \mathrm{~K}$ ). At each temperature, separate 


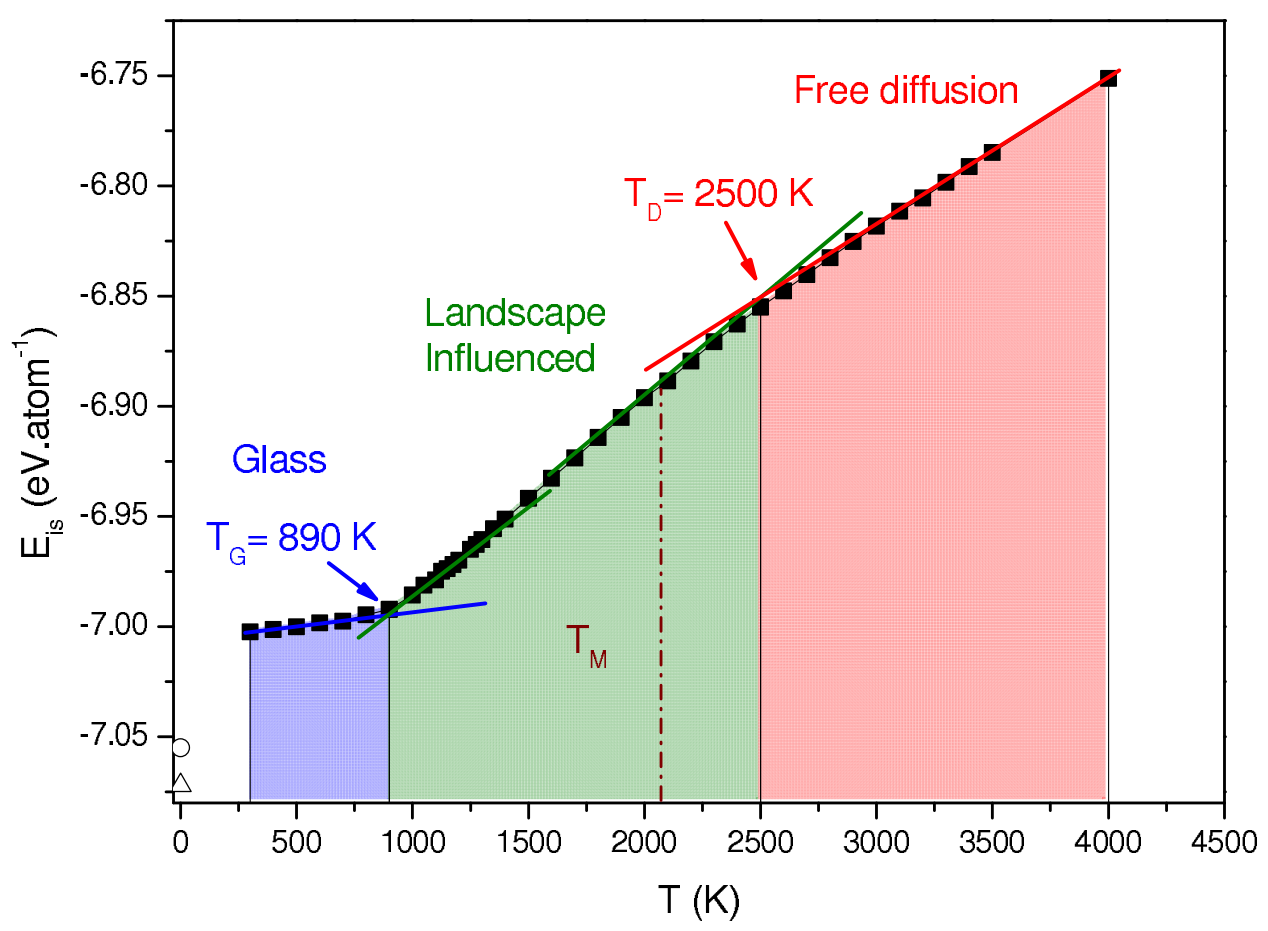

FIG. 4: Inherent-structure energy as a function of temperature for Bulk 3 system with $N=1024000$ (see text). Open symbols correspond to the ISE of the perfect crystalline phases.

production runs were performed and five independent atomic configurations were chosen in order to extract their inherent structure (IS). The IS are obtained by minimizing their energy by means of a conjugate gradient algorithm to bring the system to its local minimum in the PES, and an average value of the ISE is taken over the 5 configurations for each temperature.

In the high-temperature range corresponding to the free diffusion regime, an almost linear behavior is found. At about $T_{D}=2500 \mathrm{~K}$, the ISE starts to decrease more rapidly in the landscape influence regime. Note that the crossover $T_{D}$ occurs above the melting point $T_{M}$, which seems to be a universal feature for monoatomic liquids [77]. Finally, it is almost constant as the system, having found a deep minimum, can be considered as undergone the glass transition. From the crossover between the landscape influenced and glassy regimes[76], we estimate the glass transition temperature to be $T_{G}=890 \mathrm{~K}$ which is significantly lower than that obtained from the $H(T)$ curve shown in Fig. 3. The ratio between the melting temperature and the glass transition temperature $T_{r g}=T_{G} / T_{M}$, representing also one of the simplest glass forming ability (GFA) criterion [85], takes a value $T_{r g}=0.42$ from our simulations. 
The microscopic structural features of this glass forming ability is depicted in Fig. 5 using the common-neighbor analysis. The CNA bonds were determined from the same inherent structures from the ISE curve shown Fig. 4 using a cutoff being the first minimum of the corresponding pair-correlation function. At $T=2500 \mathrm{~K}$ the proportion of the pairs is consistent with the AIMD results [30]. The main feature emerging from Fig. 5 is an increase of the 555, 444 and 666 pairs and a decrease of the 544 and 433, representing defective 555 pairs, with decreasing temperature up to the glass transition, while the 421 and 422 pairs, correspond to the fcc and hcp structures, remain always small. The simultaneous increase of the 555 together with the decrease of the 544 and 433 indicates that the five-fold symmetry becomes better defined. Given the evolution of the amount of 433 pairs, it cannot be excluded that their decrease participates also to the increase of the 444 ones. The significant amount of 444 and 666 pairs signals the presence of bcc-like ordering. In the perfect bcc crystalline structure the ratio of the proportions of 444 to the 666 pairs is 0.75 , while the ratio observed in the simulations done here is around 0.60. This implies that a non negligible amount of 666 pairs can be associated to the 555 pairs to form Frank-Kasper (FK) polyhedra as described in details in Ref. [15]. More precisely, FK polyhedra Z14, Z15 and Z16, with 14, 15 and 16 neighbors have a specific signature in terms of the CNA: 12 pairs 555 with 2, 3 and 4 pairs 666, respectively, which can be easily detected in a simulation. A similar evolution of the basic pairs have been obtained recently by $\mathrm{Su}$ et al. [74] using an EAM potential extracted from the binary $\mathrm{Cu}-\mathrm{Zr}$ system [75]. This is also the case for the results of Mo et al. [67] mentioned above.

The fact that the coordination number of the IS evolves from 13.24 to 13.64 (from $2500 \mathrm{~K}$ to $500 \mathrm{~K}$ ) is consistent with this view. Moreover, a detail inspection of full polyhedra in a typical glass configuration $(T=300 \mathrm{~K}$ ) indicates the presence of $20186 \mathrm{Z14}, 11373 \mathrm{Z1}$, and 1793 Z16, as well as 59894 bcc polyhedra. It is worth mentioning that these polyhedra are separated and homogeneously distributed in the configuration. The simultaneous increase of FK and bcc polyhedra during cooling, and especially Z14 with the same coordination number as the bcc highlights the competition between bcc and Frank-Kasper polytetrahedral fivefold symmetry orderings and concur with the concept of geometrical frustration propounded by Tanaka $[34,35]$. 


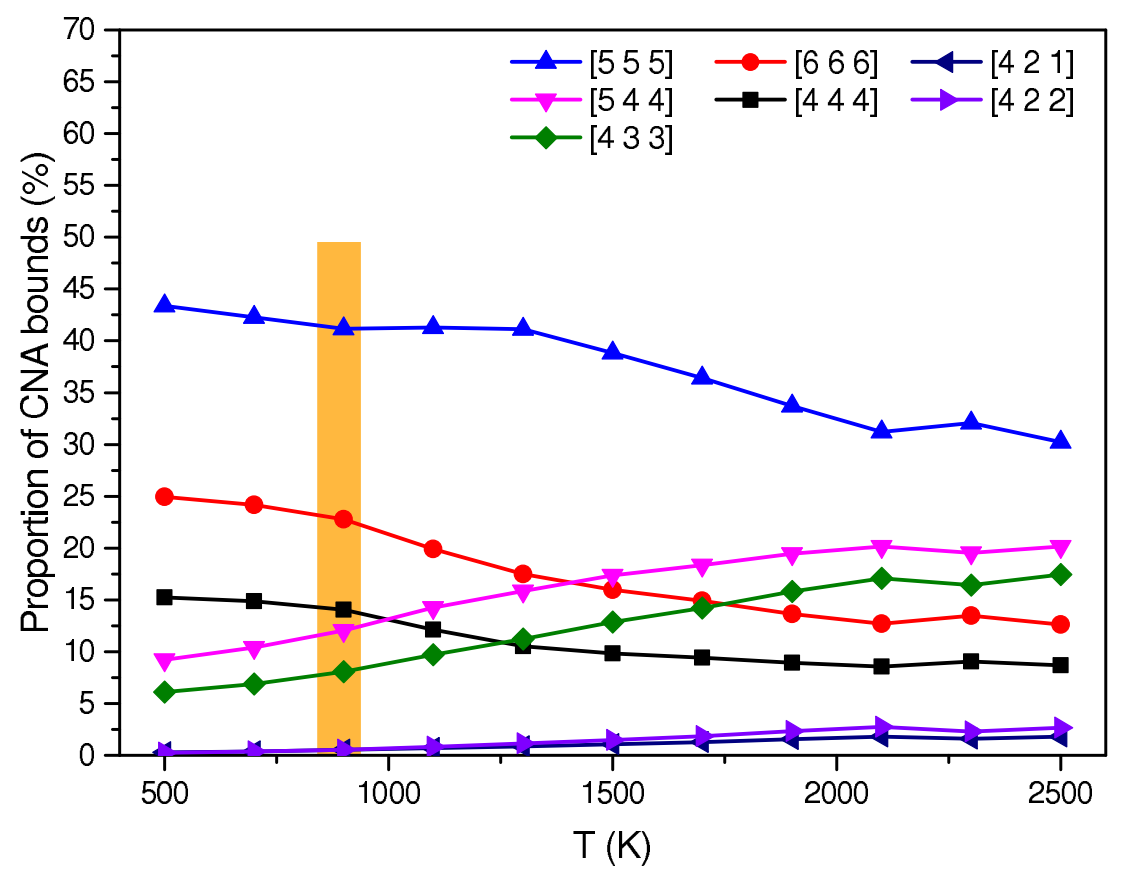

FIG. 5: Temperature evolution of the abundance of CNA bonds during the melt quenching. The vertical yellow bar indicates the glass transition region.

\section{Time-temperature-transformation curve}

To evaluate more precisely the conditions for which Zr can form a glass or crystallizes, the crystal nucleation time as a function of degree of undercooling $\Delta T$ is quantified. The resulting time-temperature-transformation curve is shown in Fig. 6. The melt quenching simulation Bulk 3 system at a cooling rate of $10^{12} \mathrm{~K} / \mathrm{s}$ was considered. For temperatures at intervals of $50 \mathrm{~K}$ in the range from $1400 \mathrm{~K}$ to $1000 \mathrm{~K}$ (as well as additional temperatures i.e. 1125,1175 et $1275 \mathrm{~K}$ ), a configuration is taken during the quench and considered as an initial configuration for an isothermal simulation at constant pressure. This range of temperatures is well below the melting temperature and just above $T_{G}$, and corresponds to deep undercooling conditions with fast homogeneous nucleation rates. At lower $\Delta T$, the nucleation time becomes large and beyond the reach for standard molecular dynamics used here. It would require advanced techniques like umbrella sampling [78], metadynamics [79], various forward-flux sampling methods [80-82], or seeding approaches[83] that will be considered elsewhere.

For each temperature, the crystal nucleation is detected by a significant drop in the potential energy with time and a threshold of $40 \%$ of the atoms having a crystalline environment 
using the CNA. The mean nucleation time for each $\Delta T$ is evaluated as the average over five independent observations, each of them being carried out with the same initial configuration but having a different random velocity distribution. The resulting TTT curve exhibits a typical nose shape with a minimum nucleation time of $0.39 \mathrm{~ns}$ around $T_{N}=1250 \mathrm{~K}$. As an important feature, the million-atom simulation cells are large enough to observe numerous nucleation events, as shown in Fig. 7. This is not always the case for Bulk 2 system with $N=16000$ atoms resulting in significant size effects, as was also pointed out very recently [26] for the study of homogeneous nucleation phenomena. As a matter of fact, repeating the simulation procedure for $N=16000$ (Bulk 2 system) leads to a TTT curve with a nose occurring at a significantly lower $\Delta T$, as can be seen in Fig. 6, and the simulation cell contains hardly more than one critical nucleus at the onset of nucleation (see Fig. 7). It is worth mentioning that, in principle, the fraction of crystalline structure follows Avrami's equation involving multiple nucleation events, which should in turn be fitted to infer the nucleation time. However, aiming to compare with Bulk 2 system, the chosen methods remains a good approximation for single nucleation events, given the fact that for such monoatomic systems nucleation is extremely rapid as can be seen in the inset of Fig. 6. This is an illustration that nucleation phenomena can hardly be treated completely and accurately with simulation box size of the order of $10^{4}$ atoms only.

\section{E. Discussion}

Renewed interests in glass formation in pure metals was recently triggered by the possibility to achieve experimentally cooling rates comparable to what is currently carried out by MD simulations [7]. The ability to form a glass is favored in systems where the melting temperature $T_{M}$ is closer to the glass transition one, $T_{G}$, as for the case of alloys with eutectic composition [1] for which it is the closest. Therefore, high values of the reduced glass $T_{r g}=T_{G} / T_{M}$ might favor glass formation. From Turnbull's observations [85], the nucleation rate maximum occurring between $T_{M}$ and $T_{G}$ is strongly correlated with $T_{r g}$. Thus it might be easier to form a glass by suppressing homogeneous nucleation for metals having high values of $T_{r g}$. For the case of $\mathrm{Zr}$ we are concerned about here, MD simulations yield a value $T_{r g}=0.42$ significantly higher that the standard rule for pure metals $T_{G} \approx 0.3 T_{M}$ [11] but obviously still lower than $T_{r g}=2 / 3$ representing a good GFA that is nearly achieved for 


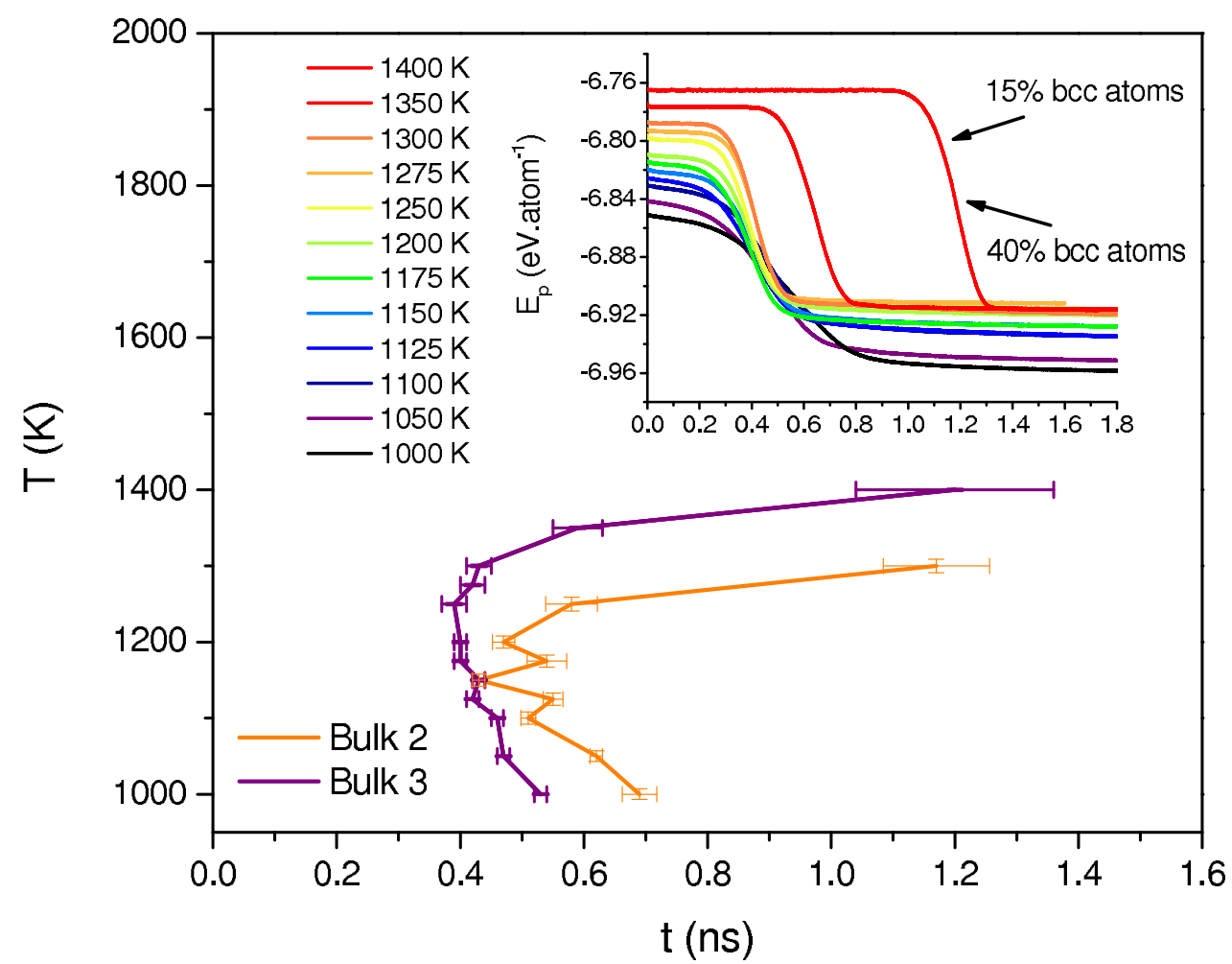

FIG. 6: Time-Temperature-transformation curve for Bulk 3 system with $N=1024000$ and Bulk 2 system with $N=16000$ in the temperature range between $T=1400 \mathrm{~K}$ and $T=1000 \mathrm{~K}$ (see text). Inset: Potential energy as a function of time for all the temperatures in the same temperature range. For each temperature, the chosen curve corresponds to the simulation in which the crystallization event occurs at a time closest to the average nucleation time. Arrows indicate the location on the potential energy curve where $15 \%$ and $40 \%$ of the atoms have a full bcc structural environment. For all temperatures $40 \%$ corresponds roughly to the half of the potential energy drop during crystallization and is taken as the measure of the nucleation time.

instance for $\mathrm{Cu}-\mathrm{Zr}$ binary alloys [86].

In recent experiments, Zhong et al. [7] showed the possibility to form a glass for pure bcc metals (Mo, V, W, and Ta) by nanosecond electrical pulses on nano-bridges achieving cooling rates as high as $10^{14} \mathrm{~K} / \mathrm{s}$. The fact that both ends of the nanobridges found to be glassy were crystalline suggests primarily that the crystal growth was suppressed. However, due to the high cooling rate and high purity of the samples, the possibility of suppressing homogeneous nucleation on the short time scale cannot either be excluded in an early stage. Focussing on pure Ta, the comparative analysis of Orava and Greer [84] reports a relatively 

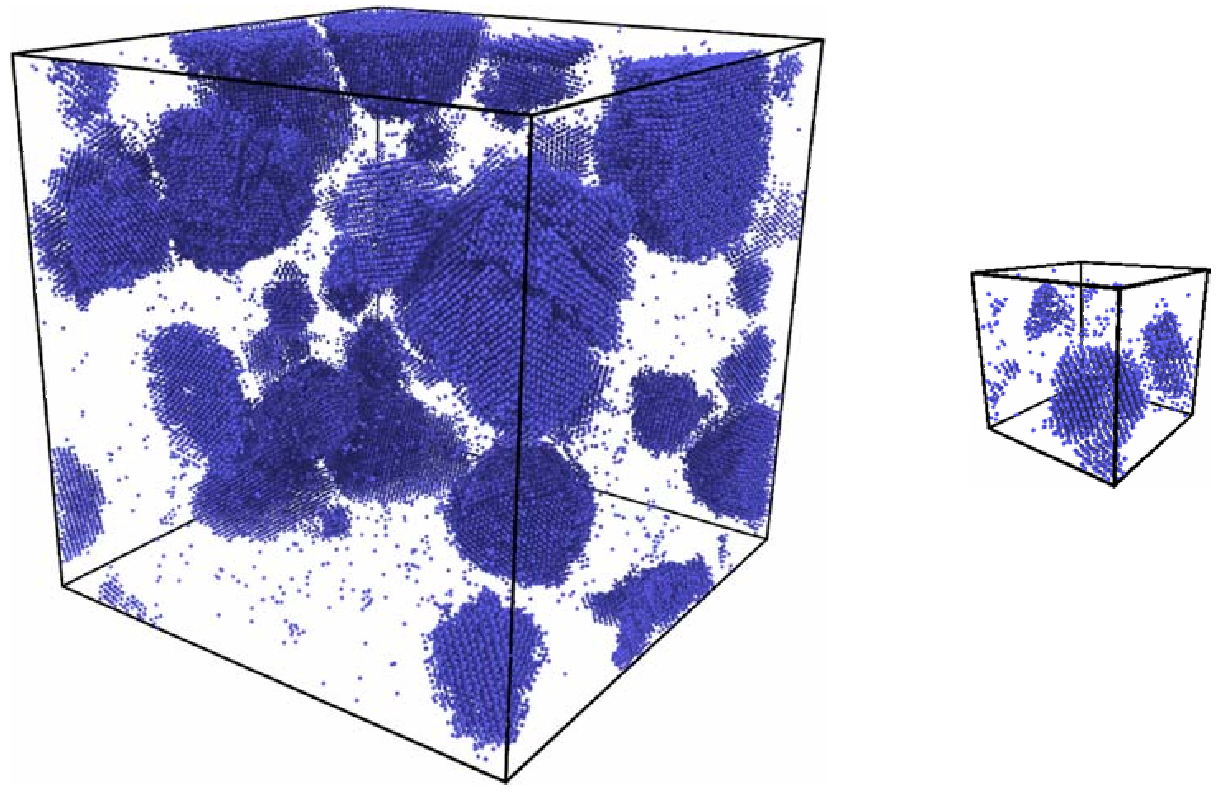

FIG. 7: Snapshot of the simulations at the onset of nucleation at $T=1150 \mathrm{~K}$ with $15 \%$ of the atoms having a bcc environment. Left panel: Bulk 3 system with $N=1024000$; Right panel: Bulk 2 system with $N=16000$. Only atoms with a bcc environment are drawn.

low value of $T_{r g}=0.34$. Nevertheless, pure Ta still shows the ability to form a glass [7] with a cooling rate of the order of a $10^{14} \mathrm{~K} / \mathrm{s}$. Interestingly, with a value of $T_{r g}=0.42$, higher than Ta, it is shown here that $\mathrm{Zr}$ can form a glass with an even lower cooling rate of $10^{12} \mathrm{~K} / \mathrm{s}$. Given the fact that the nose of the TTT curve, corresponding to the lowest nucleation time takes the value of $0.39 \mathrm{~ns}$, this cooling rate allows clearly to avoid homogeneous nucleation. Interestingly, from the nose position of the TTT curve of Bulk 3 system we obtain a critical cooling rate of $2.2 \times 10^{12} \mathrm{~K} / \mathrm{s}$, consistent with the fact that Zr crystallize during cooling with a cooling rate of $10^{11} \mathrm{~K} / \mathrm{s}$.

The first experimental attempt to form a metallic glass for Zr was done by Zhang and Zhao [36], but they retracted their paper later since what they interpreted as amorphous was in fact rapid crystal growth, and was later confirmed [37]. In their experiments, the cooling rate surely lower than the one used here might lead inevitably to crystal nucleation even if they were conducted under high pressure. Our results might also explain why Kim et al. $[5,6]$ were not able to form an amorphous with $\mathrm{Zr}$, as their cooling rate were limited to $10^{7} \mathrm{~K} / \mathrm{s}$. Nevertheless, with the possibility to reach experimentally very high cooling rates [7], the results presented here indicated that obtaining Zr metallic glass may be reachable 
experimentally.

\section{CONCLUSIONS}

In summary, a semi-empirical potential for elemental $\mathrm{Zr}$ was designed in the framework of modified embedded atom model within the second nearest neighbor formulation [46], for the purpose of performing large-scale molecular dynamics simulation of stable liquid, metastable undercooled and amorphous phases as well as homogeneous nucleation phenomena of the high temperature bcc crystalline phase. Using liquid-solid interface MD simulations, it is shown that the interaction model reproduces accurately experimental densities of the liquid and solid states in the vicinity of the melting point as well as a thermodynamic melting temperature $T_{M}=2119 \mathrm{~K}$ close to the experimental value.

The MEAM potential is able to predict dynamics properties such as the self-diffusion coefficient and the velocity auto-correlation functions as compared with ab initio molecular dynamics simulations. Furthermore, it gives a good representation of the thermodynamics properties near melting such as the enthalpy of melting of $18.2 \mathrm{~kJ} / \mathrm{mol}$ and the constant pressure specific heat of 34.4 and $34.5 \mathrm{~J} / \mathrm{mol} / \mathrm{K}$, respectively for the solid and the liquid, in good agreement with assessed experimental data.

From a melt-quenching simulation with one million atoms at a quenching rate of $10^{12}$ $\mathrm{K} / \mathrm{s}$, a glass transition temperature $T_{G}=890 \mathrm{~K}$ is observed from the evolution of the inherent structure energy, namely the crossover between the landscape-influenced and the glassy regimes of the potential-energy lanscape. It yields a glass forming ability in the sense of Turnbull $T_{r g}=T_{G} / T_{M}=0.42$ which is higher than the common rule $T_{r g} \simeq 0.3$ and similar to other bcc elemental metals [84]. The time-temperature transformation curve, determined on isotherms from initial configuration taken during the quench, displays a nose in the deep undercooling regime around $T_{N}=1250 \mathrm{~K}$ with a minimal average nucleation time of $0.39 \mathrm{~ns}$.

This finding implies the possibility for elemental Zr to form a metallic glass with cooling rates higher than $10^{12} \mathrm{~K} / \mathrm{s}$, achievable experimentally [7], and indicates that the formation of the glass might come from the avoidance of homogeneous nucleation by geometrical frustration $[34,35]$ resulting from a strong competition between bcc and fivefold FK polytetrahedral structural orderings. How this competition impacts the crystallisation kinetics still remains an open question. The present work bring the relevant computational context 
along this line of research to tackle this question in a future work.

\section{Acknowledgement}

We acknowledge the CINES and IDRIS under Project N INP2227/72914, as well as CIMENT/GRICAD for computational resources. This work was performed within the framework of the Centre of Excellence of Multifunctional Architectured Materials "CEMAM" ANR-10-LABX-44-01 funded by the "Investments for the Future" Program. This work has been partially supported by MIAI @ Grenoble Alpes, (ANR-19-P3IA-0003). Fruitful discussions within the French collaborative network in high temperature thermodynamics GDR CNRS 3584 (TherMatHT) are also acknowledged.

[1] W. Klement, R. H. Willens, P. Duwez, Nature 187, 869 (1960).

[2] H. A. Davies and J. B. Hull, J. Mater. Sci. 11, 215 (1976).

[3] W. Buckel and R. Z. Hilsch, Phys. 138, 109 (1954).

[4] K. H. Behrndt, J. Vac. Sci. Technol. 7, 385 (1970).

[5] Y. W. Kim, and T. F. Kelly, Acta Metall. Mater. 39, 3237-3249 (1991).

[6] Y. W. Kim, H. M. Lin, and T. F. Kelly, Acta Metall. Mater. 37, 247 (1989).

[7] L. Zhong, J. Wang, H. Sheng, Z. Zhang, and S. X. Mao, Nature 512, 177 (2014).

[8] A. L. Greer, Nature Mat. 14, 542 (2015).

[9] D. W. Oxtoby, J. Phys: Condens. Matter, 4, 7627 (1992).

[10] G. C. Sosso, J. Chen, S. J. Cox, M. Fitzner, P. Pedevilla, A. Zen, and A. Michaelides, Chem. Rev. 116, 7078 (2016).

[11] D. M. Herlach, S. Binder, P. Galenko, J. Gegner, D. Holland-Moritz, S. Klein, M. Kolbe, and T. Volkmann, Metall. Mater. Trans. A Phys. Metall. Mater. Sci. 46, 4921 (2015).

[12] D. Turnbull, J. Chem. Phys. 20, 411 (1952).

[13] A. Pasturel and N. Jakse, Sci. Rep. (Nature) 8, 1 (2018).

[14] F.C. Frank, Proc. Roy. Soc. London A215, 43 (1952).

[15] D. R. Nelson, F. Spaepen, in: H. Ehrenreich, F. Seitz, D. Turnbull (Eds.), Solid State Physics, 42, 1 (Academic, New York, 1989). 
[16] P. R. ten Wolde, M. J. Ruiz-Montero, and D. Frenkel, J. Chem. Phys. 104, 9932 (1996).

[17] J. H. Perepezko, Mater Sci Eng 65, 125 (1984).

[18] D. M. Herlach, Annu. Rev. Mater. Sci. 21, 23 (1991).

[19] W.-K. Rhim, S. K. Chung, D. Barber, K. F. Man, G. Gutt, A. J. Rulison, and R. E. Spjut, Rev. Sci. Instrum. 64, 2961 (1993).

[20] T. Meister, H. Werner, G. Lohoefer, D. M. Herlach, and H. Unbehauen, Eng. Pract. 11, 117 (2003).

[21] L. Hennet, V. Cristiglio, J. Kozaily, I. Pozdnyakova, H. E. Fischer, A. Bytchkov, J. W. E. Drewitt, M. Leydier, D. Thiaudière, S. Gruner, S. Brassamin, D. Zanghi, G. J. Cuello, M. Koza, S. Magazù, G. N. Greaves, and D. L. Price, Eur. Phys. J. Spec. Top. 196, 151 (2011).

[22] J. Bokeloh and G. Wilde, Jom 66, 1512 (2014).

[23] M. P. Allen, D. J. Tildesley. Computer simulation of liquids, Oxford Science Publication (1989).

[24] B. Smit, D. Frenkel. Understanding molecular simulations, 2nd ed., Academic Press, San Diego (2002).

[25] A. Mahata, M. A. Zaeem, and M. I Baskes, Modelling Simul. Mater. Sci. Eng. 26025007 (2018).

[26] A. Mahata and M. A. Zaeem, Modelling Simul. Mater. Sci. Eng. 27, 085015 (2019).

[27] H. Pang, Z. H. Jin, and K. Lu, Phys. Rev. B 67, 1 (2003).

[28] Qi An, Sheng-Nian Luo, William A. Goddard, W. Z. Han, B. Arman, and William L. Johnson, Appl. Phys. Lett. 100, 041909 (2012).

[29] Z. Y. Hou, R. S. Liu, H. R. Liu, Z. A. Tian, X. Wang, Q. Y. Zhou, and Z. H. Chen, J. Chem. Phys. 127, 174503 (2007).

[30] N. Jakse and A. Pasturel, Phys. Rev. Lett. 91195501 (2003).

[31] S. Wu, X. W. Fang, S. Y. Wang, C. Z. Wang, Y. X. Yao, K. M. Ho, Z. J. Ding, and L. Y. Chen, J. Appl. Phys. 110, 103518 (2011).

[32] N. Jakse, O. Le Bacq, and A. Pasturel, Phys. Rev. B 70, 174203 (2004).

[33] F. C. Frank and J. S. Kasper, Acta Crystallogr. 11, ibid. 184 (1958); 12, 483 (1959).

[34] H. Tanaka, Eur. Phys. J. E: Soft Matter Biol. Phys. 35, 113 (2012).

[35] J. Russo, F. Romano, and H. Tanaka, Phys. Rev. X 8, 021040 (2018).

[36] J. Zhang and Y. Zhao Nature (London) 430, 332 (2004). 
[37] T. Hattori, H. Saitoh, H. Kaneko, Y. Okajima, K. Aoki, and W. Utsumi, Phys. Rev. Lett. 96, $255504(2006)$.

[38] S. J. Plimpton, J. Comput. Phys. 117, 1 (1995), http://www.lammps.sandia.gov.

[39] M. I. Baskes, Phys. Rev. B 46, 2727 (1992).

[40] F. Willaime and C. Massobrio, Phys Rev. B 4311653 (1991).

[41] M. I. Mendelev and G. J. Ackland, Philos. Mag. Lett. 87, 349 (2007).

[42] M. I. Baskes and R. A. Johnson, Model. Simul. Mater. Sci. Eng. 2, 147 (1994).

[43] Y.-M. Kim, B.-J. Lee and M. I. Baskes, Phys. Rev. B 74, 014101 (2006).

[44] H. S. Huang, L. Q. Ai, A. C. T. van Duin, M. Chen, and Y. J. Lü, J. Chem. Phys. 151, $094503(2019)$.

[45] H. Zong, G. Pilania, X. Ding, G. J. Ackland, and T. Lookman, Npj Comput. Mater. 4, 48 (2018).

[46] B.-J. Lee, and M. I. Baskes, Phys. Rev. B 62, 8564 (2000).

[47] A. E. Gheribi, Mater. Chem. Phys. 116, 489 (2009).

[48] J. H. Rose, J. R. Smith, F. Guinea, and J. Ferrante, Phys. Rev. B 29, 2963 (1984).

[49] G. Kresse and J. Hafner, Phys. Rev. B 48, 13115 (1993).

[50] G. Kresse and J. Hafner, Phys. Rev. B 49, 14251 (1994).

[51] G. Kresse and J. Furthmüller, Comput. Mat. Sci. 6, 15 (1996).

[52] G. Kresse and D. Joubert, Phys. Rev. B, 59, 1758 (1999).

[53] J. P. Perdew, K. Burke, M. Ernzerhof, Phys. Rev. Lett. 77, 3865 (1996).

[54] J. R. Morris, C. Z. Wang, K. M. Ho, and C. T. Chan, Phys. Rev. B 49, 3109 (1994).

[55] J. R. Morris and X. Y. Song, J. Chem. Phys. 116, 9352 (2002).

[56] D. Y. Sun, M. Asta, and J. J. Hoyt, Phys. Rev. B 69, 024108 (2004).

[57] A. Stukowski, Visualization and analysis of atomistic simulation data with OVITO - the Open Visualization Tool, Modelling Simul. Mater. Sci. Eng. 18, 015012 (2010).

[58] J. D. Honeycutt, and H. C. Andersen, J. Phys. Chem. 91, 4950 (1987).

[59] D. Faken and H. Jonsson. Comput. Mat. Sci. 2. 279 (1994).

[60] T. Ishikawa, P. F. Paradis, T. Itami, and S. Yoda, Meas. Sci. Technol. 16, 443 (2005).

[61] H. P. Wang, S. J. Yang, L. Hu, and B. Wei, Chem. Phys. Lett. 653, 112 (2016).

[62] X. G. Lu, M. Selleby, and B. Sundman, Calphad Comput. Coupling Phase Diagrams Thermochem. 29, 68 (2005). 
[63] P. F. Paradis and W. K. Rhim, J. Mater. Res. 14, 3713 (1999).

[64] T. Schenk, D. Holland-Moritz,V. Simonet, R. Bellissent, and D. M. Herlach, Phys. Rev. Lett. 89, $075507(2002)$.

[65] S. Klein, D. Holland-Moritz, and D. M. Herlach, Phys. Rev. B - Condens. Matter Mater. Phys. $80,(2009)$.

[66] J. W. Arblaster, Calphad Comput. Coupling Phase Diagrams Thermochem. 43, 32 (2013).

[67] Y.-F. Mo, Z.-A. Tian, R.-S Liu, Z.-Y. Hou, L.-L. Zhou, P. Peng, H.-T Zhang, Y.-C. Liang, J. Alloys Compounds 688, 654 (2016).

[68] https://sites.google.com/site/eampotentials/Home/zr.

[69] H. W. Sheng, M. J. Kramer, A. Cadien, T. Fujita, and M. W. Chen, Phys. Rev. B 83, 134118 (2011).

[70] J. R. Fox and H. C. Andersen, J. Phys. Chem. 88, 4019 (1984).

[71] K. Vollmayr, W. Kob, and K. Binder, J. Chem. Phys. 105, 4714 (1996).

[72] S. Sastry, P. G. Debenedetti, and F. H. Stillinger, Nature 393, 554 (1998).

[73] F. H. Stillinger and T. A. Weber, Phys. Rev. A 25, 978 (1982).

[74] Y. Su a, M. Mohr, R. K. Wunderlich, X. Wang, Q. Caoa, D. Zhang, Y. Yang, H.-J. Fecht, J.-Z. Jiang, J. Molec. Liq. 298, 111992 (2020).

[75] M.I. Mendelev, M.J. Kramer, R.T. Ott, D.J. Sordelet, D. Yagodin, P. Popel, Philos. Mag. 89, 967 (2009).

[76] K. N. Lad, N. Jakse, and A. Pasturel, J. Chem. Phys. 136, 104509 (2012).

[77] F. Demmel, L. Hennet, and N. Jakse, submitted to PNAS (2020).

[78] G. M. Torrie and J. P. Valleau, J. Comput. Phys. 23, 187 (1977).

[79] A. Laio, M. Parrinello, Proc. Natl. Acad. Sci. 99, 12562 (2002).

[80] R. J. Allen, C. Valeriani, and P. Rein ten Wolde, J. Phys.: Condens. Matter. 21, 463102 (2009).

[81] P. G. A. Bolhuis, D. Chandler, C. Dellago, and P. L. Geissler, Annu. Rev. Phys. Chem. 53, $291(2002)$.

[82] T. S. van Erp, D. Moroni, and P. G. A. Bolhuis, J. Chem. Phys. 118, 7762 (2003).

[83] V. G. Baidakov, K. S.Bobrov, and A. S. Teterin, J. Chem. Phys. 135, 054512 (2011).

[84] J. Orava and A. L. Greer, J. Chem. Phys. 140, 214504 (2014).

[85] D. Turnbull, Contemp. Phys. 10, 473 (1969). 
[86] Q. Wang, L.-M. Wang, M. Z. Ma, K. Binder, T. Volkmann, D. M. Herlach, J. S. Wang, Q. G. Xue, Y. J. Tian, and R. P. Liu, Phys. Rev. B 83, 014202 (2011). 